statistically significant results, suggestive of increased risks. Recently, the follow-up in the cohort was extended. We report on a case-based study to investigate these suggestions.

Methods From the extended follow-up, cases of breast, stomach and lung cancer in women, and of brain cancer in men were identified. It was planned that the lung and breast cancer cases would be interviewed and compared with matched controls, and that the rarer stomach and brain cancers would be examined case-only. A questionnaire was designed to collect detailed employment histories within the factory and elsewhere, information relevant to possible asbestos exposure, and lifestyle and environmental factors. A historical hygiene assessment was carried out at the factory, to inform construction of a job-exposure matrix.

Results Attempts to recruit cases (or proxy respondents) were only partially successful; as a result, the lung cancers element was converted to a case-only study. Comparison with controls was possible for only 20 breast cancer cases (including 7 proxy respondents). From an extensive programme of conditional logistic analyses, statistical significance was achieved for exposure to arsenic compounds, antimony trioxide and sulphuric acid mist and to gases in general, but only in a few of the analyses. Examination of proxy responses for stomach, lung and brain cancers did not suggest any common workplace factors for any of these outcomes.

Conclusions We interpreted this evidence as not supportive of an occupational risk for any of the cancers.

\title{
Poster-discussion: Cancer
}

P153 CASE-CONTROL AND CASE-ONLY STUDIES OF SELECTED CANCERS AT A SCOTTISH SEMICONDUCTOR MANUFACTURING FACILITY

Brian Miller, Laura MacCalman, ${ }^{1}$ Karen Galea, ${ }^{1}$ Sam Wilkinson, ${ }^{2}$ John Cherrie, ${ }^{1}$ Andrew Darnton, ${ }^{2}$ Amy Shafrir, ${ }^{1}$ Damien McElvenny, ${ }^{3}$ John Osman² $1 / 0 M$, Edinburgh, UK; ${ }^{2}$ Health \& Safety Executive, Bootle, UK; ${ }^{3}$ Statistics \& Health Ltd, Manchester, UK

10.1136/oemed-2011-100382.367

Objectives HSE's 2001 report on cancer in a Scottish cohort of semiconductor manufacturing workers showed some 